\title{
Nonlinear Dynamics of Torsion Lattices
}

\author{
V. V.Smirnov, M. A. Kovaleva, L. I. Manevitch
}

We present an analysis of torsion oscillations in quasi-one-dimensional lattices with periodic potentials of the nearest neighbor interaction. A one-dimensional chain of point dipoles (spins) under an external field and without the latter is the simplest realization of such a system. We obtained dispersion relations for the nonlinear normal modes for a wide range of oscillation amplitudes and wave numbers. The features of the short wavelength part of the spectrum at large-amplitude oscillations are discussed. The problem of localized excitations near the edges of the spectrum is studied by the asymptotic method. We show that the localized oscillations (breathers) appear near the long wavelength edge, while the short wavelength edge of the spectrum contains only dark solitons. The continuum limit of the dynamic equations leads to a generalization of the nonlinear Schrödinger equation and can be considered as a complex representation of the sine-Gordon equation.

Keywords: essentially nonlinear systems, coupled pendulums, nonlinear normal modes, limiting phase trajectories

Received December 12, 2017

Accepted February 28, 2018

The reported study was supported by the Russian Science Foundation according to the research projects 16-13-10302.

Valery V.Smirnov

vvs@polymer . chph .ras.ru

Margarita A. Kovaleva

margo.kovaleva@gmail.com

Leonid I. Manevitch

manevitchleonid3@gmail.com

Semenov Institute of Chemical Physics RAS

ul. Kosygina 4, Moscow, 119991 Russia

RUSSIAN JOURNAL OF NONLINEAR DYNAMICS, 2018, 14(2), 179-193 


\section{Introduction}

The phonons are the key objects for various processes in solids. They are responsible for heat transfer, thermal expansion and diffusion. Their interaction with charge carriers in quite a number of cases controls the conductivity of solids. The description of phonon dynamics in nanostructures [1] is close to the fundamental theses of solid state theory due to the original soundness of the crystal lattice and the absence of grain boundaries at the nanoscale. Therefore, the nanoscale objects serve as a perfect proving ground for testing the phonon theory, in particular, for large-amplitude oscillations. The normal oscillations of the crystal lattice are usually considered in small-amplitude approximations because any collective motions of atoms with amplitudes which exceed several per cent of the interatomic distances lead to the melting or the destruction of the crystal lattice. However, some molecular crystals admit torsion oscillations with large amplitudes (up to the full rotation) without any violation of the crystal order. The normal paraffins (n-alkanes) in the crystal state show a transition into the so-called "rotational" phase at temperatures close to the melting point [2]. In such a phase the alkane chain rotates around its axis as a whole. Obviously, in such a case we cannot restrict our consideration to small-amplitude oscillations. So the problem of torsion oscillations turns out to be essentially nonlinear, even if we do not take into account the internal torsion of the chain's fragments. The oscillations of the paraffin chain as a whole in the force field of the molecular environment lead to the problem of the oscillations of a rigid pendulum, the "gravitational" force of which is determined by interaction with the other chains in the crystal. However, since the ground state of the paraffin chain is a plane trans-zigzag conformation, some internal torsions of the chain fragments are possible. This degree of freedom is controlled by the internal torsion/conformational potential, which is a periodic function of the rotation angle of the neighbor chain's fragments [3].

Another example of the torsion oscillations corresponds to the chain of dipoles. Actually, the interaction between the point-like dipoles (spins) is determined by the difference of the rotation angles of the dipoles. If the external field is absent, the dynamics of the dipole chain is controlled by this interaction, which is a periodic function of the above-mentioned difference. The long wavelength approximation, which is the conventional approach for the analysis of such systems leads to linearization of the internal interaction. As a result, we get the well-known Frenkel-Kontorova model [4], the continuum version of which is the sine-Gordon equation [5]. The properties and solutions of the latter are well-studied $[4,5]$, but it does not allow us to analyze the short wavelength excitations in discrete systems.

The main goal of the paper is to analyze nonlinear normal modes, in particular, in the large-amplitude limit as well as the possibility of the existence of localized modes near the edges of the spectrum for discrete systems with periodic-type interparticle interactions.

\section{Torsion nonlinear normal modes}

The energy of the interaction of the one-dimensional system of point-like dipoles (spins) can be written as follows:

$$
E=\sum_{j=1}^{N}\left(D_{j} D_{j+1}+D_{j} E_{e x}\right),
$$

where $D_{j}$ and $E_{e x}$ are the dipole moments and the external field intensity, respectively, and $N$ is the chain length. Let us measure the dipole's rotation angle as the angle between the direction of the external filed and the dipole. In such a case the first term in Eq. (2.1) is the 
function of the difference between the dipole's rotation angles and the second term depends on the rotation angle itself:

$$
E \sim \sum_{j=1}^{N}\left(D^{2} \cos \left(q_{j+1}-q_{j}\right)+E_{e x} D \cos q_{j}\right) .
$$

The ground state of system (2.2) is controlled by the relation between the energies of the dipole-dipole interaction and the interaction of the dipole and the external field. If $\left|E_{e x}\right|<D_{j}$, the ground state is determined by the relation $q_{j+1}-q_{j}=\pi$. Therefore, it is useful to introduce the modulation function $\varphi_{j}=(-1)^{j} q j$ and to set the origin with zero value of the ground state. This choice corresponds to the Hamiltonian function of the system as follows:

$$
H=\sum_{j}\left(\frac{1}{2}\left(\frac{d \varphi_{j}}{d t}\right)^{2}+\left(1-\cos \left(\varphi_{j+1}-\varphi_{j}\right)\right)+\sigma\left(1-\cos \varphi_{j}\right)\right)
$$

with $\varphi_{j}=0(j=1, \ldots, N)$ for the coordinates of the ground state. (One should note that the dimensionless time $t$ in the Hamiltonian function (2.3) is normalized with the velocity of the long wavelength oscillations, while the lattice constant is taken to be unity.) We keep the parameter $\sigma$ for the interaction between the dipole and the external field in order to generalize the system to a wider class of physical objects (spin chains, molecules and crystals of flexible polymers, etc.) $[6,7]$.

The equations of motion of system (2.3) are well known:

$$
\frac{d^{2} \varphi_{j}}{d t^{2}}-\sin \left(\varphi_{j+1}-\varphi_{j}\right)+\sin \left(\varphi_{j}-\varphi_{j-1}\right)+\sigma \sin \varphi_{j}=0
$$

The long wavelength approximation assumes that the differences between the rotation angles of the nearest dipoles are small: $\varphi_{j+1}-\varphi_{j} \ll 1$. Under this assumption the internal interaction terms in Eqs. (2.4) can be linearized:

$$
\frac{d^{2} \varphi_{j}}{d t^{2}}-\varphi_{j+1}+2 \varphi_{j}-\varphi_{j-1}+\sigma \sin \varphi_{j}=0
$$

Equations (2.5) are the known equations of the Frenkel - Kontorova model [4], the continuum analogue of which is the sine-Gordon equation [5]. The latter admits partial solutions such as the localized $2 \pi$-rotation of the chain (kinks) and the localized oscillations (breathers). The solutions describing the long wavelength oscillations can be found clearly. However, there is no general theory that would allow us to study both the extended and localized solutions of the discrete system (2.4).

In order to analyze Eqs. (2.4), we introduce new variables

$$
\Psi=\frac{1}{\sqrt{2}}\left(\frac{i}{\sqrt{\omega}} \frac{d \varphi}{d t}+\sqrt{\omega} \varphi\right)
$$

where $\omega$ is a still undefined frequency. The inverse transformation can be written as

$$
\varphi=\frac{1}{\sqrt{2 \omega}}\left(\Psi+\Psi^{*}\right), \quad \frac{d \varphi}{d t}=-i \sqrt{\frac{\omega}{2}}\left(\Psi-\Psi^{*}\right),
$$

where the asterisk $\left.{ }^{*}\right)$ denotes the complex conjugation. 
Substituting expressions (2.7) into Eqs. (2.4) and expanding the trigonometric functions into series, we get:

$$
\begin{aligned}
& i \frac{d}{d t} \Psi_{j}-\frac{\omega}{2}\left(\Psi_{j}-\Psi_{j}^{*}\right)- \\
& -\frac{1}{\sqrt{2 \omega}} \sum_{k} \frac{(-1)^{k}}{(2 k+1) !}\left(\frac{1}{\sqrt{2 \omega}}\right)^{2 k+1}\left[\left(\Psi_{j+1}-\Psi_{j}+\text { c.c. }\right)^{2 k+1}-\right. \\
& \left.-\left(\Psi_{j}-\Psi_{j-1}+\text { c.c. }\right)^{2 k+1}-\left(\Psi_{j}+\Psi_{j}^{*}\right)^{2 k+1}\right]=0,
\end{aligned}
$$

where "c.c." replaces the complex conjugated terms.

Let us consider the single-frequency solutions of Eqs. (2.8):

$$
\Psi_{j}=\psi_{j} e^{-i \omega t},
$$

where $\psi_{j}$ do not depend on the time variable $t$.

Substituting these expressions into Eq. (2.8) and multiplying the latter by factor $e^{i \omega t}$, after averaging over the period we get the system of stationary equations for the values $\psi_{j}$ :

$$
\begin{gathered}
\frac{\omega \psi_{j}}{2}-\frac{1}{\sqrt{2 \omega}}\left[J_{1}\left(\sqrt{\frac{2}{\omega}}\left|\psi_{j+1}-\psi_{j}\right|\right) \frac{\psi_{j+1}-\psi_{j}}{\left|\psi_{j+1}-\psi_{j}\right|}-\right. \\
\left.-J_{1}\left(\sqrt{\frac{2}{\omega}}\left|\psi_{j}-\psi_{j-1}\right|\right) \frac{\psi_{j}-\psi_{j-1}}{\left|\psi_{j}-\psi_{j-1}\right|}+\sigma J_{1}\left(\sqrt{\frac{2}{\omega}}\left|\psi_{j}\right|\right) \frac{\psi_{j}}{\left|\psi_{j}\right|}\right]=0,
\end{gathered}
$$

where $J_{1}$ is the Bessel function of the first order.

It is easy to see that the values $\psi_{j}=\sqrt{X} e^{i \kappa j}$ (with $X=$ const) are the solutions of Eqs. (2.10) if the relation

$$
\frac{\omega}{2} \sqrt{X}-\frac{1}{\sqrt{2 \omega}}\left(2 J_{1}\left(2 \sqrt{\frac{2 X}{\omega}} \sin \frac{\kappa}{2}\right) \sin \frac{\kappa}{2}+\sigma J_{1}\left(\sqrt{\frac{2 X}{\omega}}\right)\right)=0
$$

is valid.

Equation (2.11) determines the frequency of the normal oscillations as a function of the absolute value of the complex variable $\Psi$ and the wave number $\kappa$. At first sight, Eq. (2.11) is intricate enough for finding the frequencies of the normal oscillations. However, the relationship between the absolute value of the function $|\Psi|=\sqrt{X}$ and oscillation amplitude $Q$, which follows from expression (2.6)

$$
X=\frac{\omega}{2} Q^{2},
$$

drastically simplifies Eq. (2.11). Taking into account the latter, the explicit form of the eigenfrequency can be written as follows:

$$
\omega^{2}=\frac{2}{Q}\left(2 J_{1}\left(2 Q \sin \frac{\kappa}{2}\right) \sin \frac{\kappa}{2}+\sigma J_{1}(Q)\right) .
$$

One should note that no assumptions concerning the values of the oscillation amplitude $Q$ and the wave number $\kappa$ have been made in order to get expression (2.13). Therefore, one should assume that the expression obtained will be valid for a wide range of values. 
It is obvious that the long wavelength $(\kappa \ll 1)$ and small-amplitude $(Q \ll 1)$ limit of relation (2.13) leads to the dispersion relation of the linear problem: $\omega \sim \sqrt{\sigma+4 \sin ^{2} \kappa / 2}$. Figure 1 shows the dispersion relation (2.13) for different values of the oscillation amplitudes. The left panel (a) demonstrates the latter for the chain without any external field, while the right panel (b) shows the effect of the external field on the dynamics of the chain.
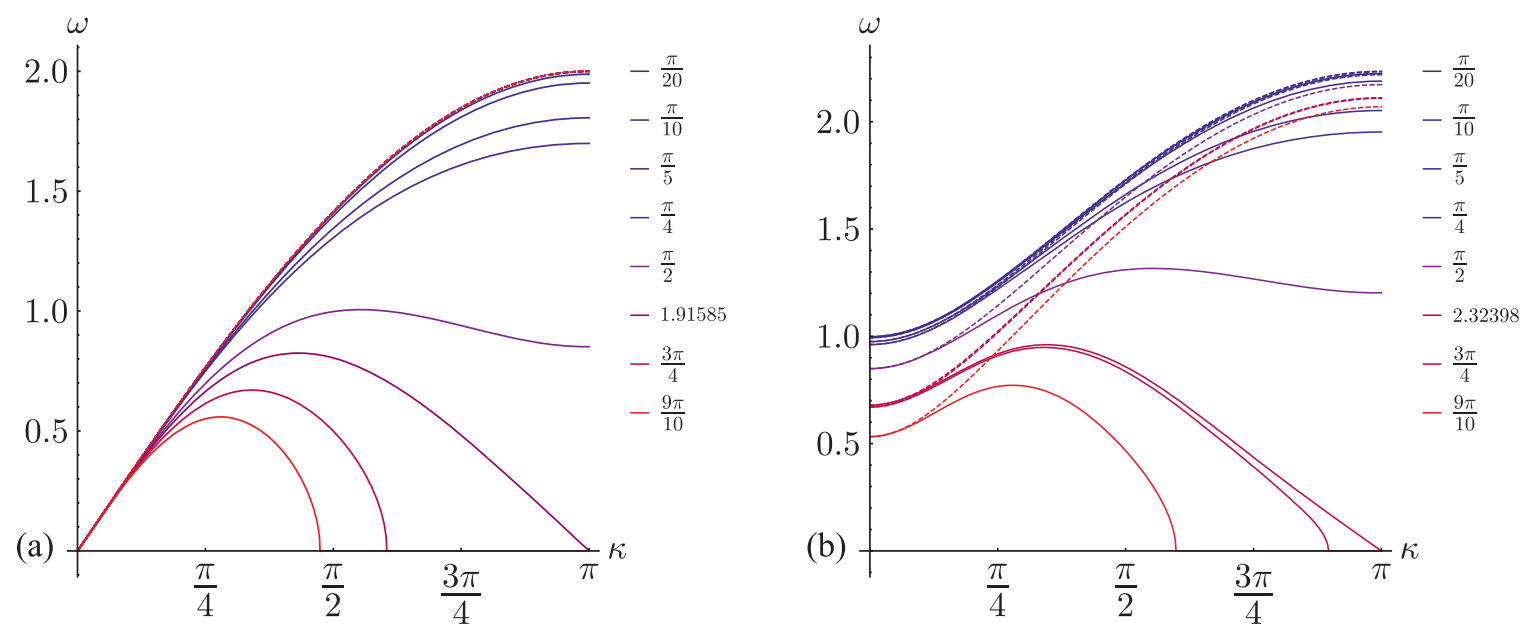

Fig. 1. Dispersion relations (2.13) for the chain without (a) and under the influence of the external field at different oscillation amplitudes (the values are shown on the right of the panels). The amplitude values where the frequency of the $\pi$-mode is equal to zero $(\omega(\kappa=\pi)=0)$ are shown in the decimal format. The intensity of the external field is $\sigma=1$. The dispersion relations for the Frenkel-Kontorova model are shown in the right panel by the dashed curves. The respective relation for the linear problem without any external field is shown by the dashed curve in the left panel.

The dispersion relations shown in Fig. 1 are in good agreement with the known relations for the Frenkel - Kontorova model and for the chain with a linear potentials in the small-amplitude limit. However, if the oscillation amplitudes are large enough, the differences from the abovementioned models become drastic.

First of all, the gap frequency $\omega_{0}=\omega(\kappa=0)$ in the presence of the external field decreases, while the oscillation amplitude grows. It is an ex ante result, because the uniform oscillations of the chain are analogous to the oscillations of the pendulum, the frequency of which tends to zero as $Q \rightarrow \pi$. Comparison of the gap frequency $\omega_{0}=\sqrt{2 J_{1}(Q) / Q}$ with exact value $\pi / 2 K(\sin Q / 2)$ ( $K$ is a complete elliptic integral of the first kind) [10] for the frequency of the pendulum oscillations indicates the boundaries of the validity of the approach considered. The estimation of the gap frequency shows that it is in good agreement with the exact value up to amplitude $Q \sim 7 \pi / 10$ (the discrepancies do not exceed $10 \%$ ), however, the value of $\omega_{0}$ is not equal to zero at $Q=\pi$. The observed divergence is explained by the fact that the averaging procedure applied by us is not adequate for the oscillation amplitudes $Q \sim \pi$ due to the presence of a large number of multiple resonances, which should be taken into account when the secular terms are extracted in Eq. (2.11).

The second essential feature of the dispersion relation (2.13) is that the frequency of the antiphase oscillations $\left(\pi\right.$-mode, $\varphi_{j+1}=-\varphi_{j}$ ) vanishes near the oscillation amplitude $Q \sim 3 \pi / 4$. This results in the appearance of forbidden bands in the spectrum. The source of such a behavior is that the force between the nearest dipoles becomes repulsive when the difference of the rotation angles exceeds $\pi / 2$. This can lead to the onset of the rotation mobility in the conservative system 
similar to (2.3). However, the presence of a weak dissipation (which is not considered here) may be a reason for the stabilization of the oscillations. The value of the antiphase oscillations amplitude with frequency $\omega(\kappa=\pi)=0$ can be estimated as the root of the equation:

$$
2 J_{1}(2 Q)+\sigma J_{1}(Q)=0 .
$$

The fact that this value exceeds the amplitude $Q=\pi / 2$ (for $\sigma=0$, see Fig. 1a), also follows from the underestimation of the multiple resonances.

Concluding this section, one can say that the nonlinear normal modes of the discrete system and their frequencies are studied for a wide range of oscillation amplitudes and wave numbers.

The next section is concerned with analysis of the nonstationary dynamics of the system near the edges of the spectrum.

\section{Nonstationary dynamics: the interaction of the nonlinear normal modes}

The essential difference between the linear and nonlinear normal modes consists in the possibility of the effective interaction of the latter if their frequencies are close. As a result, the intricate dynamics of the nonlinear systems cannot be represented as a sum of different oscillations, but leads to a new form of the system's motion including localized modes and chaotic regimes [10]. In this section we will obtain nonstationary equations which describe the resonantly interacting nonlinear modes in the framework of the approach based on Eqs. (2.8)(2.10).

In order to do it, one should note that Eqs. (2.10) can be considered as equilibrium conditions for the system with the energy

$$
H_{r}=\sum_{j=1}^{N}\left(\frac{\omega}{2}\left|\psi_{j}\right|^{2}+J_{0}\left(\sqrt{\frac{2}{\omega}}\left|\psi_{j+1}-\psi_{j}\right|\right)+\sigma J_{0}\left(\sqrt{\frac{2}{\omega}}\left|\psi_{j}\right|\right)\right),
$$

where $J_{0}$ is the Bessel function of zero order.

Considering the function $H_{r}$ as the Hamiltonian function of some system, one can get the equations of motion according to the rules:

$$
i \frac{\partial \psi_{j}}{\partial \tau}=-\frac{\partial H_{r}}{\partial \psi_{j}^{*}}
$$

The latter leads to the equations

$$
\begin{gathered}
i \frac{\partial \psi_{j}}{\partial \tau}+\frac{\omega \psi_{j}}{2}-\frac{1}{\sqrt{2 \omega}}\left[J_{1}\left(\sqrt{\frac{2}{\omega}}\left|\psi_{j+1}-\psi_{j}\right|\right) \frac{\psi_{j+1}-\psi_{j}}{\left|\psi_{j+1}-\psi_{j}\right|}-\right. \\
\left.-J_{1}\left(\sqrt{\frac{2}{\omega}}\left|\psi_{j}-\psi_{j-1}\right|\right) \frac{\psi_{j}-\psi_{j-1}}{\left|\psi_{j}-\psi_{j-1}\right|}+\sigma J_{1}\left(\sqrt{\frac{2}{\omega}}\left|\psi_{j}\right|\right) \frac{\psi_{j}}{\left|\psi_{j}\right|}\right]=0 .
\end{gathered}
$$

At this point the questions arise: (i) what kind of the system motion is described by Eqs. (3.2) and (ii) what is time " $\tau$ "?

We have already shown that some functions $\psi_{j}$ can be the solutions of Eqs. (3.2) if $\partial \psi_{j} / \partial \tau=$ $=0$. On the other hand, the motions with the specific time $T \sim 1 / \omega$ have been excluded by 
the choice of functions $\Psi_{j}$ (Eq. (2.9)) and the consequent averaging. So, if we assume the parameter $\omega=$ const, Eqs. (3.2) will describe the motions, which can be distinguished from the stationary solution only slightly. They may be nonlinear normal modes, the parameters of which (the oscillation amplitude or the wave number) are close to the stationary solution, or the combinations of such modes. It is important to understand that the specific time scale for the variable " $\tau$ " is controlled by the remoteness $\delta \omega$ of the nonstationary solution from the stationary one in the "frequency" space. So, Eqs. (3.2) describe the motions with the slow time $\tau \sim(\delta \omega / \omega) t$. In such a case the stationary solution corresponding to the nonlinear normal mode with the frequency $\omega$ is imaged as the stationary point in the phase space of system (3.1).

Summarizing the above, we conclude that the solutions of Eqs. (3.2) should be considered as combinations of nonlinear normal modes with close frequencies. Such resonant conditions can occur for small discrete systems in the small-amplitude limit near the edges of the spectrum: at $\kappa \ll 1$ for the chain under the influence of the external field and at $\kappa \sim \pi$ for the systems in the presence and without any external field. While the chain length grows, resonant interactions can also be observed for the oscillations with a large amplitude and the very different wave numbers (see, for example, the dispersion relation for oscillations with the amplitude $Q=\pi / 2$ in Fig. 1b). However, the most intriguing are the combinations of the nonlinear normal modes with the close wave numbers, because they result in essentially nonuniform distributions of the energy along the chain $[8,9]$. It was shown that the description of the resonant dynamics of the chain in terms of the normal modes is noneffective [8]. Instead of the modes we introduce new variables which should describe the energy distribution rather than the dipoles motion.

For the normal modes near the spectrum edges such variables are represented as linear combinations of the modes. Each of the new variables corresponds to some domain of the chain inside which the dipoles are moved coherently, while their behavior in the different domains differs essentially. Therefore, these new variables have been called "the coordinates of the coherent domains". We define the coordinates of the coherent domains as follows:

$$
\begin{aligned}
& \chi_{1}(\tau)=\frac{1}{\sqrt{2 N}} \sum_{j} \psi_{j}(\tau)\left(1+\cos \left(\kappa j+\frac{\pi}{4}\right)\right), \\
& \chi_{2}(\tau)=\frac{1}{\sqrt{2 N}} \sum_{j} \psi_{j}(\tau)\left(1-\cos \left(\kappa j+\frac{\pi}{4}\right)\right),
\end{aligned}
$$

where $\kappa=\frac{2 \pi}{N}$ and the phase shift $\pi / 4$ has been added in order to place the domains' centers at the points $j=N / 4$ and $j=3 N / 4$. It can be shown that for the limiting case $N=2$ the domain coordinates transform to the coordinates of the particles. The inverse transformation to the functions $\psi_{j}$ is defined as follows:

$$
\psi_{j}(\tau)=\frac{1}{\sqrt{2 N}}\left(\left(\chi_{1}(\tau)+\chi_{2}(\tau)\right)+\left(\chi_{1}(\tau)-\chi_{2}(\tau)\right) \cos \left(\kappa j+\frac{\pi}{4}\right)\right) .
$$

In addition to the energy integral (3.1), the system (3.2) admits an extra integral of motion, which is an analogue of the quantum-mechanical occupation number:

$$
X=\sum_{j}\left|\psi_{j}\right|^{2}
$$

Transformation (3.3) keeps both integrals of the motion:

$$
X=\left|\chi_{1}\right|^{2}+\left|\chi_{2}\right|^{2} .
$$


This allows us to study the nonstationary dynamics more effectively. Taking into account relation (3.6), one can write the domain coordinates in the polar representation:

$$
\chi_{1}=\sqrt{X} \cos \theta e^{i \delta_{1}}, \quad \chi_{2}=\sqrt{X} \sin \theta e^{i \delta_{2}} .
$$

One should notice that parameter $X$ determines the excitation of the system as a whole, while the value $R=\left|\chi_{1}\right|^{2}-\left|\chi_{2}\right|^{2}=\cos 2 \theta$ shows the difference in the domain occupations. The latter is changed from 1 to -1 with variation of $\theta$ from 0 up to $\pi / 2$.

Substituting expressions (3.4), (3.3), (3.7) into the Hamiltonian function (3.1), we find that the energy of the system is a function of the relative amplitude $\theta$ and the phase difference $\Delta=\delta_{1}-\delta_{2}$. This fact converts the phase space of the system into a two-dimensional one and the dynamics of the system can be imaged on the phase plane $(\Delta, \theta)$.

The general representation of the energy in the polar variables $\theta$ and $\Delta$ is intricate enough, but it admits an effective analysis near the spectrum edges.

\section{Nonstationary dynamics of the edge modes}

The spectrum of the normal oscillations in the discrete system under periodic boundary conditions consists of normal modes with the wave numbers $\kappa=2 \pi k / N, k=0,1, \ldots, N-1$, where $N$ is the number of particles in the chain. However, the spectrum of the linear system is twice degenerate and only the wave numbers $k \leqslant N / 2$ are sensible. As mentioned above, the resonant conditions appear near the spectrum edges $(\kappa \ll 1$ and $\kappa \sim \pi)$, which is obvious from expression (2.13):

$$
\omega \sim \omega_{\text {edge }} \pm \text { const }\left(\frac{\Delta \kappa}{2}\right)^{2},
$$

where the sign $(+/-)$ corresponds to the left and right spectrum edges, respectively, and $\Delta \kappa \sim 2 \pi / N$.

As shown in $[8,9]$, resonant interactions of the normal modes near the spectrum edges lead to an effective energy capture in one of the coherent domains with the subsequent localization of oscillations, i.e., the formation of breathers. We will analyze these conditions for the system under study.

\subsection{Long wavelength limit: $\kappa \ll 1$}

It follows from Fig. 1b that, under the influence of the external field in the small-amplitude long wavelength limit, the torsion lattices are similar to the Frenkel-Kontorova model. The respective conditions of the resonant interaction and the transition to the localization of the oscillations have been studied in [9] for the left edge of the spectrum.

The value of the gap frequency follows from expression (2.13):

$$
\omega_{0}=\sqrt{\frac{2}{Q} J_{1}(Q)} .
$$

Assuming that $\kappa=2 \pi / N$ is small enough for the resonance to occur, we express the domain coordinates (3.4) in terms of the polar variables $\theta$ and $\Delta$ and write the Hamiltonian function (3.1) as

$$
\left.H=\frac{\omega_{0}^{2} Q^{2}}{4}+\sum_{j=1, \ldots, N}\left(J_{0}\left(Q \sqrt{\frac{2}{N} r_{1}(\theta, \Delta)}\right)\right)+\sigma J_{0}\left(Q \sqrt{\frac{1}{2 N} r_{2}(\theta, \Delta)}\right)\right),
$$


where

$$
\begin{aligned}
& r_{1}=(1-\cos \Delta \sin 2 \theta)(1-\sin (2 j+1) \kappa) \sin ^{2} \frac{\kappa}{2} \\
& r_{2}=2\left(1+\sqrt{2} \cos 2 \theta \sin \left(\kappa j+\frac{\pi}{4}\right)\right)+(1-\cos \Delta \sin 2 \theta) \sin 2 \kappa j .
\end{aligned}
$$

Expression (4.1) allows us to analyze the domains' occupation $R$ for different values of the oscillation amplitude $Q$ on the phase plane $\{R, \Delta\}$ (Fig. 2).
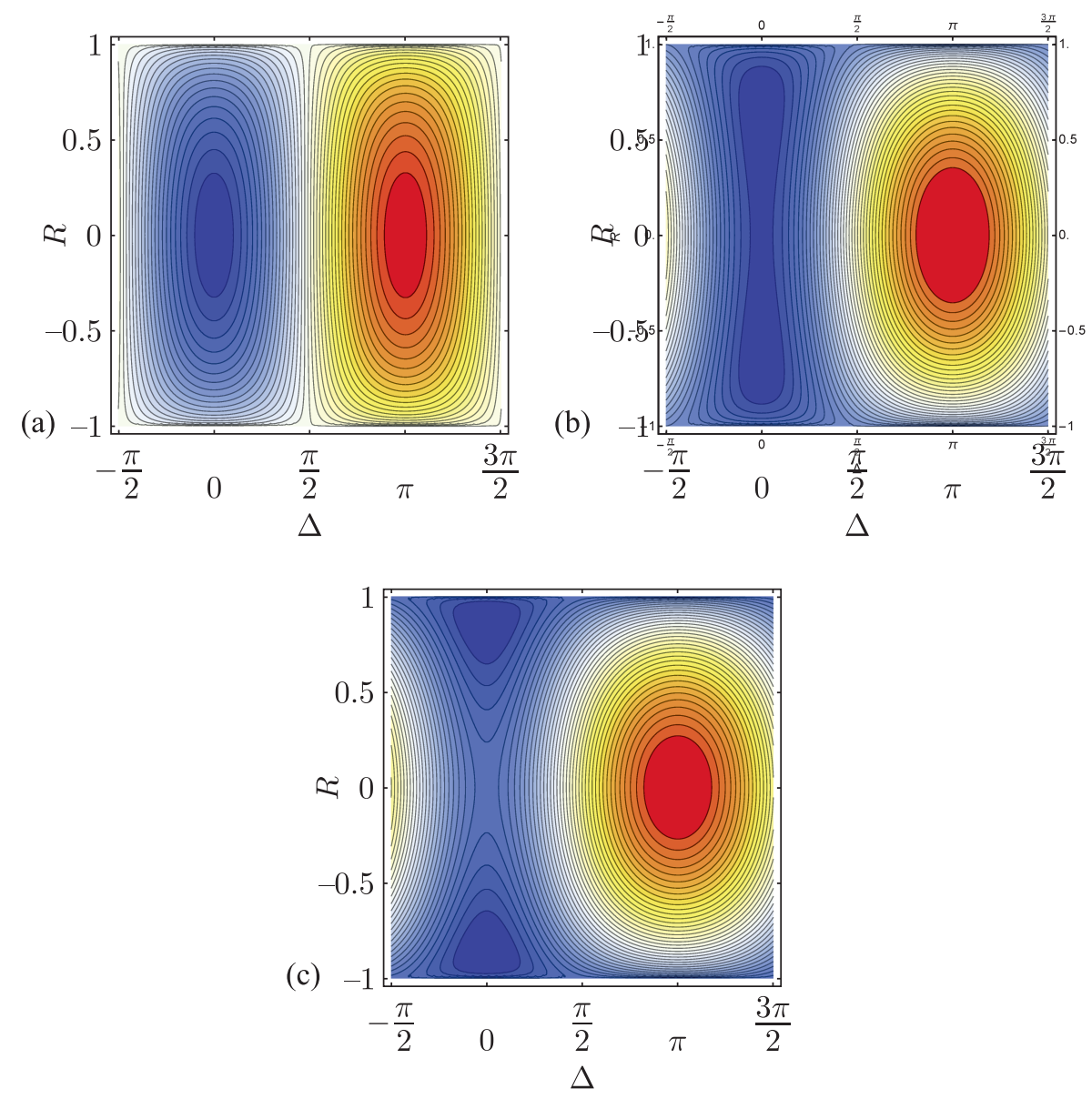

Fig. 2. Phase planes $\{R, \Delta\}$ of system (3.1) at various values of the oscillation amplitudes $Q$. The chain length $N=32$. (a) before the instability bifurcation $Q=2 \pi / 10$, (b) after instability bifurcation, but before the localization $Q=0.8 \pi$, (c) after localization bifurcation $Q=0.96 \pi$.

First of all, one should remember that the nonlinear normal modes correspond to the stationary points on the phase plane $\{R, \Delta\}$. The coordinates of the stationary points are: $(R=0, \Delta=0)$ for the gap mode and $(R=0, \Delta=\pi)$ for the next one. The coherent domains correspond to the lines $R= \pm 1$. All the trajectories surrounding the stationary points describe a slow redistribution of the energy between the coherent domains. This redistribution may be complete or partial depending on the remoteness of the trajectory from the stationary point. Only the trajectory passing through the "pure" domain states $R= \pm 1$ leads to the complete energy exchange between the domains. It is called the limiting phase trajectory (LPT) as it is the most remote from the stationary points and it separates the phase plane into quadrants, each of which contains the normal mode. 
The analysis of the phase plane shows that the shape of the LPT is close to rectangular at a small oscillation amplitude (see Fig. 2a) and the stationary points are stable. The time evolution of the LPT is well described by the saw-tooth function. While the oscillation amplitude grows, the gap mode loses its stability and two new stationary states arise (Fig. 2b). The threshold of the instability $Q_{\text {inst }}$ can be estimated by the equation

$$
\left.\frac{\partial^{2} H}{\partial \theta^{2}}\right|_{(\theta=\pi / 4 ; \Delta=0)}=0 \text {. }
$$

Two new stationary points correspond to the weakly nonuniform distribution of the energy between the coherent domains. However, the process of complete energy exchange is still possible along the LPT. While the oscillation amplitude grows, the nonuniformity of the energy distribution increases. The separatrix passing through the unstable gap mode expands and it coincides with the LPT when the energies of the gap mode and the "pure" domain states become equal (Fig. 2c). The respective value of the oscillation amplitude can be calculated as the solution of the equation

$$
H\left(\theta=\frac{\pi}{4}, \Delta=0\right)=H(\theta=0, \Delta=\pi / 2) .
$$

For the oscillations with the amplitude, which exceeds the localization threshold $Q_{l o c}$, there is no trajectory passing from the state $R=-1$ to the state $R=+1$ and vice versa. Therefore, the oscillation energy turns out to be localized in the initially excited region of the chain (domain). Figure 3 shows the variation of the instability and localization thresholds with the chain length.

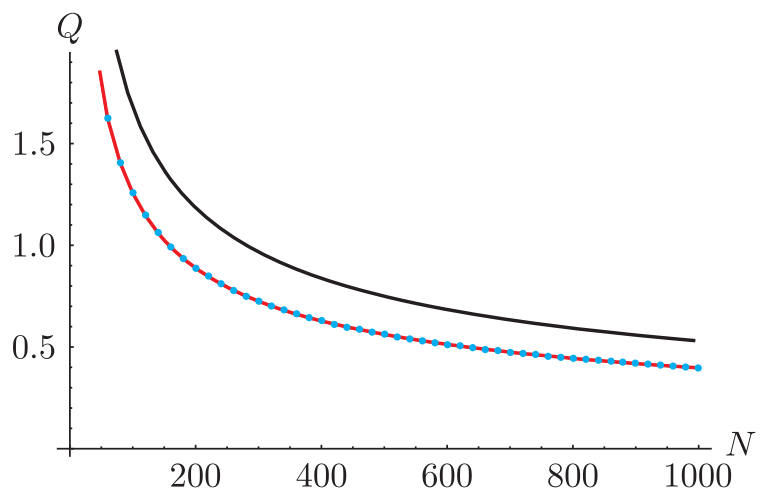

Fig. 3. The values of the instability (solid red curve) and localization (black) thresholds vs the chain length. The points show the approximation of the instability threshold $Q_{\text {inst }}$ by the expression $Q=$ $=$ const $/ \sqrt{N}$ with const $\sim 12.5$.

Figure 3 shows that the dependence of the instability threshold $Q_{\text {inst }}$ on the chain length is well approximated by $N^{-1 / 2}$, which is in good agreement with the respective value for the Fermi-Pasta - Ulam lattice, where the analogous phenomenon occurs in the vicinity of the short wavelength edge of the spectrum $[8,12,13]$.

If the chain length $N \rightarrow \infty$, the frequency difference $\delta \omega \rightarrow 0$ and the analysis performed above is not valid since the resonant conditions are realized for some set of the modes. In such a case one should consider the continualization of Eq. (3.2).

$$
i \frac{\partial \psi}{\partial \tau}+\frac{\omega}{2} \psi+\frac{1}{2 \omega} \frac{\partial^{2} \psi}{\partial x^{2}}-\frac{\sigma}{\sqrt{2 \omega}} J_{1}\left(\sqrt{\frac{2}{\omega}}|\psi|\right) \frac{\psi}{|\psi|}=0 .
$$


Equation (4.4) is the generalization of the well-known nonlinear Schrödinger equation (NLSE) and its small amplitude limit can be written as follows:

$$
i \frac{\partial \psi}{\partial \tau}+\frac{\omega^{2}-\sigma}{2 \omega} \psi+\frac{1}{2 \omega} \frac{\partial^{2} \psi}{\partial x^{2}}+\frac{\sigma}{8 \omega^{2}}|\psi|^{2} \psi=0 .
$$

Equation (4.5) is known as the "focusing" NLSE and admits the localized excitations (breathers) as the partial solution [14].

So, one can say that the analysis performed above shows the possibility of energy localization in the discrete torsion lattices near the long wavelength edge of the spectrum and allows us to determine the threshold amplitude of the localization. The continual version of the equations of motion (4.4) can be considered as the complex representation of the sine-Gordon equation.

\subsection{Short wavelength limit: $\kappa \sim \pi$}

The largest wave number $\kappa=\pi$ corresponds to the antiphase oscillations ( $\pi$-mode, $\psi_{j+1}=-\psi_{j}$ ) of the chain with the frequency

$$
\omega=\sqrt{\frac{2}{Q}\left(2 J_{1}(2 Q)+\sigma J_{1}(Q)\right)} .
$$

In order to analyze such oscillations, it is useful to introduce the modulating function $\phi_{j}=(-1)^{j} \psi_{j}$. In such a case Eqs. (3.2) can be written as follows:

$$
\begin{gathered}
i \frac{\partial \phi_{j}}{\partial \tau}+\frac{\omega \phi_{j}}{2}-\frac{1}{\sqrt{2 \omega}}\left[J_{1}\left(\sqrt{\frac{2}{\omega}}\left|\phi_{j+1}+\phi_{j}\right|\right) \frac{\phi_{j+1}+\phi_{j}}{\left|\phi_{j+1}+\phi_{j}\right|}+\right. \\
\left.+J_{1}\left(\sqrt{\frac{2}{\omega}}\left|\phi_{j}+\phi_{j-1}\right|\right) \frac{\phi_{j}+\phi_{j-1}}{\left|\phi_{j}+\phi_{j-1}\right|}+\sigma J_{1}\left(\sqrt{\frac{2}{\omega}}\left|\phi_{j}\right|\right) \frac{\phi_{j}}{\left|\phi_{j}\right|}\right]=0 .
\end{gathered}
$$

The respective dispersion relation becomes:

$$
\omega^{2}=\frac{2}{Q}\left(2 J_{1}\left(2 Q \cos \frac{\kappa}{2}\right) \cos \frac{\kappa}{2}+\sigma J_{1}(Q)\right) .
$$

It was shown that the resonant interaction near the right edge of the spectrum in the FermiPasta-Ulam lattices occurs in accordance with the scenario discussed above [8]. However, in contrast to the FPU-chain, the torsion lattices are specified by the soft nonlinearity. Therefore, the existence of the localized solutions (breathers) near the high-frequency edge of the spectrum is not obvious.

Let us define the domain variable according to expressions (3.4), (3.3). Then the Hamiltonian function (3.1) of the system should be written as follows:

$$
H_{r}=\frac{\omega^{2}}{4} Q^{2}+\sum_{j=1, \ldots, N}\left[J_{0}\left(Q \sqrt{\frac{2}{N} r_{1}(\theta, \Delta)}\right)+\sigma J_{0}\left(Q \sqrt{\frac{1}{N} r_{2}(\theta, \Delta}\right)\right],
$$

where the functions $r_{1}, r_{2}$ are

$$
\begin{aligned}
r_{1}(\theta, \Delta) & =3+4 \sqrt{2} \cos 2 \theta \cos \frac{\kappa}{2} \sin \left(\frac{\pi}{4}+\frac{2 j+1}{2} \kappa\right)+\cos \kappa(1+\sin (2 j+1) \kappa)+ \\
& +2 \sin (2 j+1) \kappa+\cos \Delta \sin 2 \theta\left(1-\cos \kappa-2 \cos ^{2} \frac{\kappa}{2} \sin (2 j+1) \kappa\right), \\
r_{2}(\theta, \Delta) & =2+2 \cos 2 \theta(\cos \kappa j+\sin \kappa j)+1-\sin 2 \theta \cos \Delta \sin \kappa j .
\end{aligned}
$$



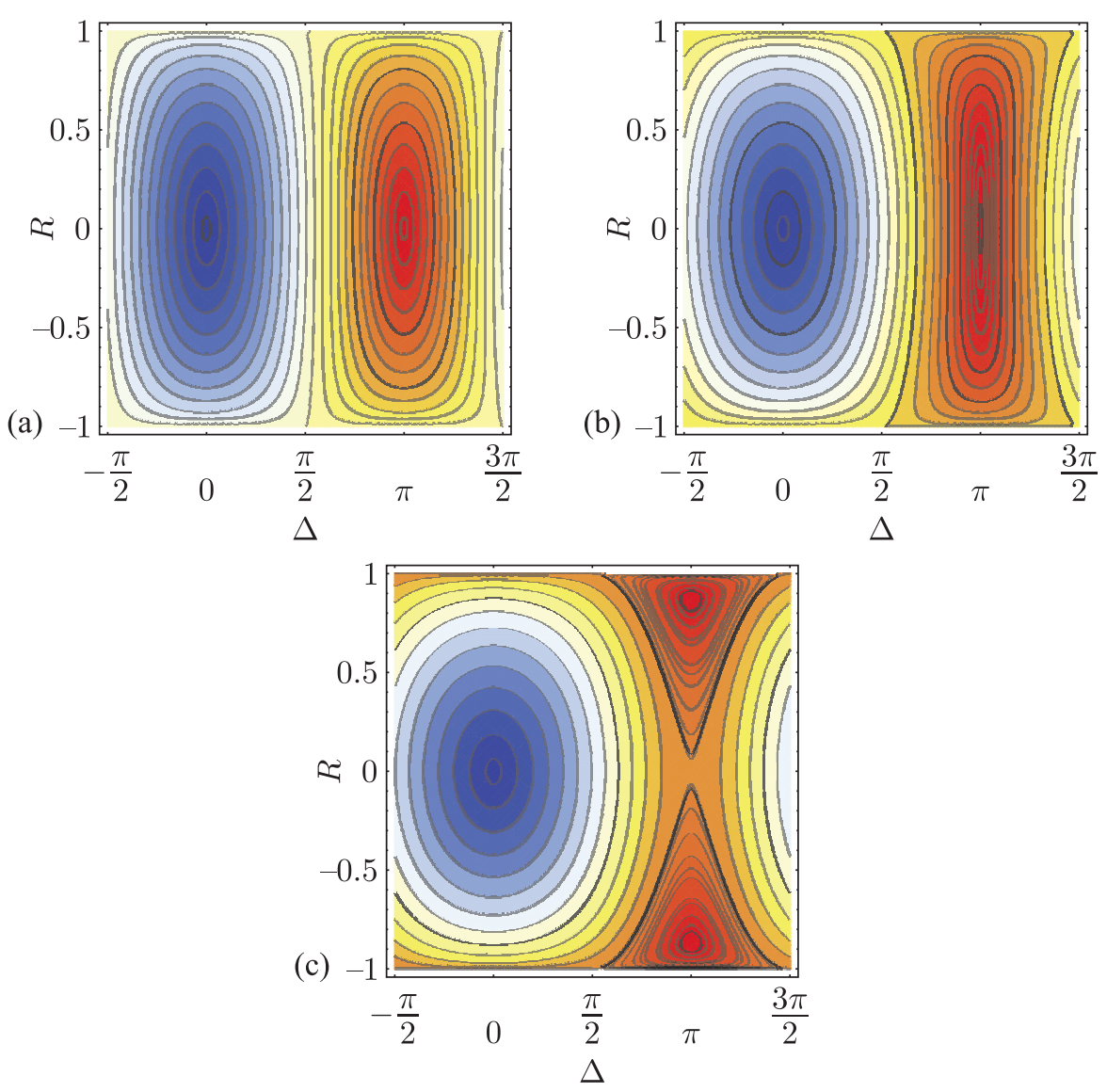

Fig. 4. Phase portraits of system (4.9) at the oscillation amplitudes $Q=\pi / 10$ (a), $Q=2 \pi / 10$ (b), $Q=3.2 \pi / 10$ (c). The external field intensity $\sigma=1$ and the chain length $N=32$.

Taking into account that $R=\cos 2 \theta$, we consider the phase portraits as was done above. Figures $4 \mathrm{a}-4 \mathrm{c}$ show the phase portraits for various values of the oscillation amplitude $Q$.

Comparison of Figs. 2 and 4 shows that the topology of the phase space is similar in the small-amplitude limit. However, for the short wavelength edge (Fig. 4) the zone-bounding $\pi$-mode turns out to be stable in the whole range of the oscillation amplitudes. The bifurcations, which lead to the loss of stability and the following localization of the trajectories, occur for the mode which is the closest to the $\pi$-mode. Such a coalition does not lead to energy localization like breathers, but gives rise to the dark solitons $[9,15]$. One should notice that the "bright" and "dark" solitons in the small system are very similar due to a weak localization of the oscillations [9], but they are absolutely different in the case of the infinite chain. The reasons for the nonoccurrence of the breathers near the high-frequency edge of the spectrum are that the oscillation frequencies decrease with growing amplitude. Therefore, the breather's frequency is found inside the phonon band. Only the discrete breathers, the frequency of which exceeds the phonon's frequencies essentially, are possible in the torsion lattices [11].

Figure 5 shows the dependences of the instability and localization thresholds on the chain length. One should notice that these values are essentially lower than the respective values for the left edge of the spectrum and the effect of the external field is practically negligible. Nevertheless, the above-mentioned dependences are well approximated by the same function $Q \sim 1 / \sqrt{N}$ (see Fig. 5). 


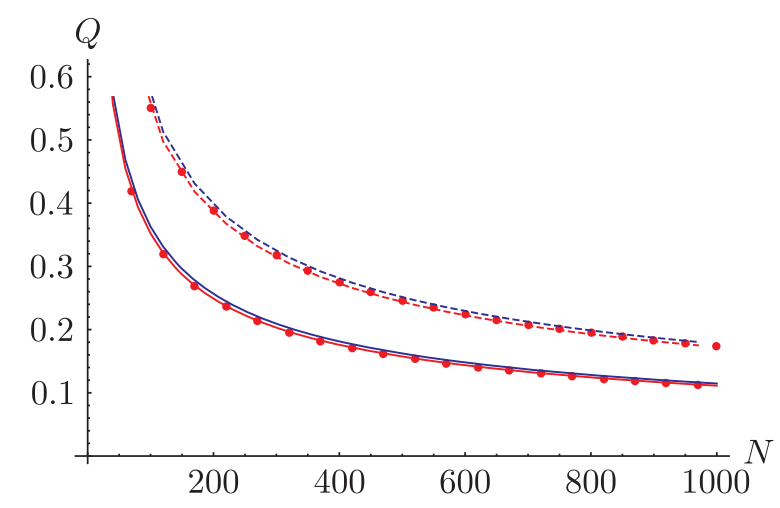

Fig. 5. Instability and "localization" thresholds vs the chain length. Solid blue and red show the instability thresholds for the intensity of the external field $\sigma=0$ and $\sigma=1$, respectively. The dashed curves represent the "localization2" thresholds. The dots show the approximate values $Q \sim \operatorname{const} / \sqrt{N}$ with the constants 3.5 and 5.5 , respectively.

$R$ and $\Delta$ make up the pair of conjugated variables for the Hamiltonian function (4.9) and the equations of motion can be obtained in accordance with the relations

$$
\frac{\partial R}{\partial \tau}=\frac{\partial H}{\partial \Delta}, \quad \frac{\partial \Delta}{\partial \tau}=-\frac{\partial H}{\partial R} .
$$

The equations obtained can be integrated numerically for the various values of the oscillation amplitudes. Figure 6 represents the data of such integrations for the lattice with $N=32$ under periodic boundary conditions. The initial conditions have been chosen as the extremely close to the LPT. The black and blue curves show the domains occupation (right panel) and the phase shift (left panel) for the oscillation amplitude, which are smaller than the instability threshold. The red curves correspond to the initial amplitude which exceeds the localization threshold approximately by $10 \%$.

Analyzing Fig. 6, one should pay attention to the strong variation of the period of the energy exchange (see also Fig. 7). In the linear system the simple combination of two close normal modes leads to the slow migration of the oscillation energy along the chain (beating) with the specific time, which is determined by the difference between the modes' frequencies.
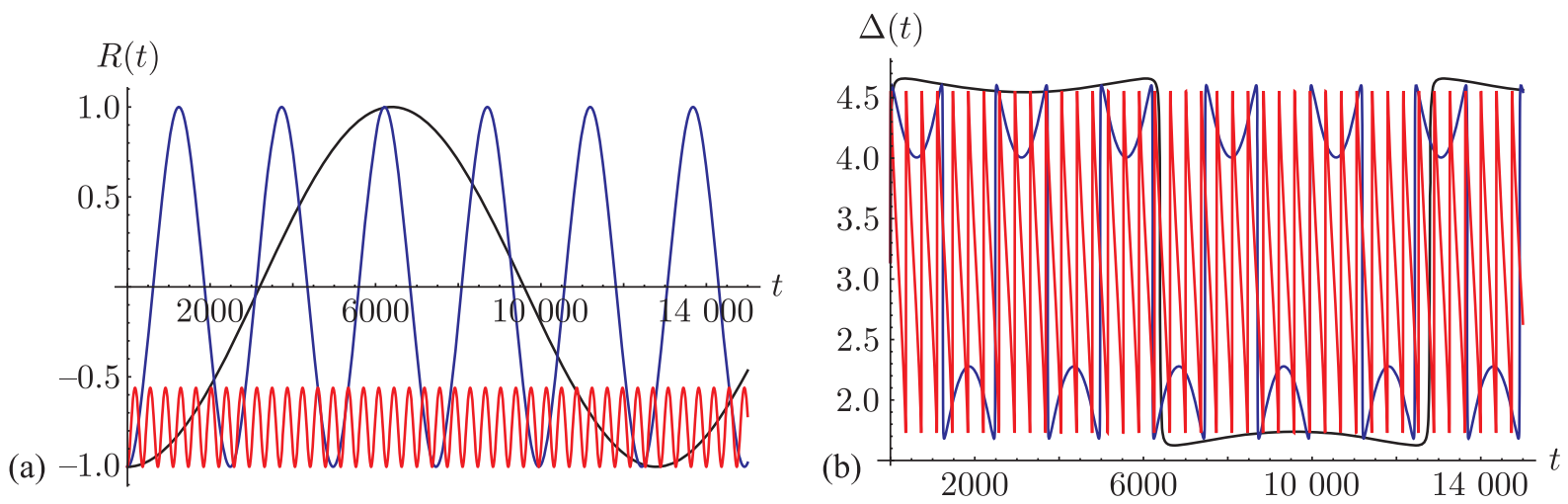

Fig. 6. Difference of the domains' occupation $R(\tau)$ (a) and phase shift $\Delta(\tau)$ (b) vs time at three oscillation amplitudes: $Q=0.1 \pi<Q_{\text {inst }}$ (black curves), $Q_{\text {inst }}<Q=0.2 \pi<Q_{\text {loc }}$ (blue curves) and $Q=$ $=0.32 \pi>Q_{\text {loc }}$ (red curves). $N=32, \sigma=1$. Initial conditions: $R(0)=-0.9999, \Delta(0)=\pi$. 


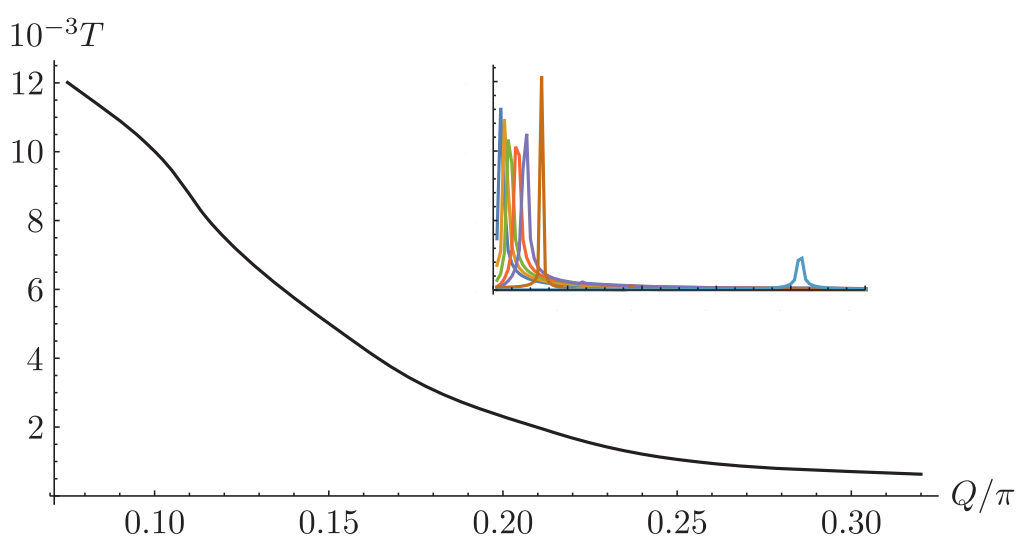

Fig. 7. Period of the energy exchange vs oscillation amplitude. The insert shows the Fourier-spectra. Intensity of the external field $\sigma=1$, chain length $N=32$. Initial conditions: $R(0)=-0.9999, \Delta(0)=\pi$.

In the nonlinear system the period of the beating depends on the amplitude, but its variation should not exceed approximately 20 per cent of the initial magnitude for the oscillations shown in Fig. 6. However, one can see in Fig. 7 that the period changes by a factor of more than 6 . It is the consequence of the strong nonlinear interaction of the modes.

\section{Conclusion}

The analysis of the torsion lattices performed above allows us to reveal the essential peculiarities of the torsion dynamics of discrete systems at large amplitudes of oscillations. The analytical representation for the nonlinear normal modes and the frequencies of the normal oscillations have been obtained for the lattices under the effect of the external field and without it. The decrease of the oscillation frequencies with growing amplitude leads to the formation of a forbidden band, where the normal modes cannot exist. The analytical results are in good agreement with the data of the numerical simulation of the torsion lattices of various lengths.

We have studied the nonlinear interaction of the normal modes near the edges of the spectrum of discrete systems. In the long wavelength limit this interaction leads to the localization of the oscillation energy in some domain of the chain if the oscillation amplitude exceeds some threshold value. In the chain of the infinite length such a capture of the oscillations leads to the creation of breathers. The nonlinear Schrödinger equation for the chain of the infinite length has been obtained as the continuum limit of the discrete system equations. It can be considered as a complex representation of the sine-Gordon equation. The interaction of the nonlinear modes near the short wavelength of the spectrum results in the creation of the "dark" localization because of the "soft" nonlinearity of the system. Due to the latter the frequency of the localized mode is in the phonon band and the bright solitons (breathers) can effectively interact with the normal oscillations of the lattice.

One should mention that the analysis performed above allows us to reveal the peculiarities of the torsion dynamics, which can be significant from the viewpoint of understanding the physical processes taking place in the dipole (spin) lattices, single molecules and crystals of the flexible polymers and biopolymers. The modern nanotechnologies are based on the fundamental properties of the single molecules and nanostructures. Therefore, the results obtained above can give very useful information for the development of the nano-electro-mechanical resonators and other devices. 


\section{References}

[1] Stroscio, M. and Dutta, M., Phonons in Nanostructures, Cambridge: Cambridge Univ. Press, 2001.

[2] Mukherjee, P. K., Phase Transitions among the Rotator Phases of the Normal Alkanes: A Review, Phys. Rep., 2015, vol. 588, pp. 1-54.

[3] Volkenstein, V.M., Configuration Statistics of Polymeric Chains, Moscow: Akad. Nauk, 1959 (Russian).

[4] Braun, O.M. and Kivshar, Yu.S., The Frenkel-Kontorova Model: Concepts, Methods, and Applications, Berlin: Springer, 2004.

[5] The sine-Gordon Model and Its Applications: From Pendula and Josephson Junctions to Gravity and High-Energy Physics, J. Cuevas-Maraver, P. Kevrekidis, F. Williams (Eds.), Cham: Springer, 2014.

[6] Takeno, Sh. and Homma, Sh., A sine-Lattice (sine-Form Discrete sine-Gordon) Equation: One- and Two-Kink Solutions and Physical Models, J. Phys. Soc. Japan, 1986, vol. 55, no. 1, pp. 65-75.

[7] Yomosa, S., Soliton Excitations in Deoxyribonucleic Acid (DNA) Double Helices, Phys. Rev. A, 1983, vol. 27, no. 4, pp. 2120-2125.

[8] Manevitch, L. I. and Smirnov, V. V., Limiting Phase Trajectories and the Origin of Energy Localization in Nonlinear Oscillatory Chains, Phys. Rev. E, 2010, vol. 82, no. 3, 036602, 9 pp.

[9] Smirnov, V. V. and Manevich, L. I., Limiting Phase Trajectories and Dynamic Transitions in Nonlinear Periodic Systems, Acoust. Phys., 2011, vol. 57, no. 2, pp. 271-276.

[10] Sagdeev, R. Z., Usikov, D. A., and Zaslavsky, G. M., Nonlinear Physics: From the Pendulum to Turbulence and Chaos, Chur: Harwood Acad. Publ., 1990.

[11] Takeno, Sh. and Peyrard, M., Nonlinear Rotating Modes: Green's-Function Solution, Phys. Rev. E, 1997, vol. 55, no. 2, pp. 1922-1928.

[12] Dauxois, Th. and Peyrard, M., Physics of Solitons, Cambridge: Cambridge Univ. Press, 2010.

[13] Lichtenberg A.,Livi R., Pettini M., Ruffo S. Dynamics of Oscillator Chains. in The Fermi-PastaUlam Problem: A Status Report, G. Gallavotti (Ed.), Lect. Notes Phys., vol. 728, Berlin: Springer, 2008, pp. 21-121.

[14] Scott, A., Nonlinear Science: Emergence and Dynamics of Coherent Structures, 2nd ed., New York: Oxford Univ. Press, 2003.

[15] Kivshar, Yu. S. and Luther-Davies, B., Dark Optical Solitons: Physics and Applications, Phys. Rep., 1998, vol. 298, nos. 2-3, pp. 81-197. 